\title{
Beyond Cellular Green Generation: Potential and Challenges of the Network Separation
}

\author{
Ilario Filippini, Alessandro Enrico Cesare Redondi, and Antonio Capone \\ Dipartimento di Elettronica, Informazione e Bioingegneria, Politecnico di Milano, Milan, Italy \\ Correspondence should be addressed to Ilario Filippini; ilario.filippini@polimi.it
}

Received 7 September 2016; Accepted 28 November 2016; Published 13 February 2017

Academic Editor: María Calderon

Copyright (C) 2017 Ilario Filippini et al. This is an open access article distributed under the Creative Commons Attribution License, which permits unrestricted use, distribution, and reproduction in any medium, provided the original work is properly cited.

\begin{abstract}
This article introduces the ideas investigated in the $\mathrm{BCG}^{2}$ project of the GreenTouch consortium. The basic concept is to separate signaling and data in the wireless access network. Transmitting the signaling information separately maintains coverage even when the whole data network is adapted to the current load situation. Such network-wide adaptation can power down base stations when no data transmission is needed and, thus, promises a tremendous increase in energy efficiency. We highlight the advantages of the separation approach and discuss technical challenges opening new research directions. Moreover, we propose two analytical models to assess the potential energy efficiency improvement of the $\mathrm{BCG}^{2}$ approach.
\end{abstract}

\section{Introduction}

In the last few years, the explosive evolution of the mobile data demand is stressing operators' networks, which need to upgrade the capacity provided to the users. Predictions are that, over the next 10 years, the traffic volume will grow by more than a factor of 30 and the number of connected devices to many billions. Facing this growth, mobile operators are challenged with higher costs and $\mathrm{CO} 2$ footprint in providing more capacity, with flattening revenues. This requires mobile networks to become ever more cost- and resource- efficient.

Besides CAPEX considerations, operational costs are becoming an issue to be carefully addressed. It is well-known that radio access nodes are particularly energy-hungry, as their energy consumption can reach more than the $80 \%$ of the total energy in the entire access network. This motivated research in the field of green wireless networking where the main aim is to develop devices, design protocols, and find network planning and optimization strategies that include energy efficiency aspects in the normal network operation. Energy saving mechanisms are now available in many commercial products as well. The green networking community has addressed the energy efficiency issue in communications following three main tracks: (i) improving radio access nodes, (ii) improving network deployments, and (iii) changing the system management.
Activities within the first group identified a huge potential for energy savings in the power amplifiers, for example, [1], by introducing new classes of power amplifiers, such as the class $\mathrm{S}$ amplifiers. More energy can be saved by spending less power on unused wireless resources, for example, by muting unused time slots and frequencies. In [2] the authors find that up to $97 \%$ of the wireless resources in OFDM systems are unused in access cells. A similar approach has been proposed for W-CDMA systems in [3] by muting $5 \mathrm{MHz}$ bands. Research activities within the second track, that is, network deployments, exploit that shorter links can be operated at lower signal strengths. This significantly decreases the power spent at the device amplifiers. Most approaches decrease cell size, for example, to micro- and femtocells, while aiming for the optimal cell size, for example, [4]. The third group, finally, performs network-wide management by switching to sleep mode unnecessary access devices, for example, when there are no active users to serve or if users can be served by other cells [5]. This approach can be applied to the wired part of the access network as well, providing further energy savings [6]. Since solving the network-wide optimization problem on the fly is computationally too complex, for example, [7-9], mainly heuristics are proposed to decide which cell to be put in sleep mode, for example, [10-12].

From analyzing the first group of approaches, it can be concluded that improving the energy efficiency of devices will 
already do a very good job in reducing the overall energy consumption of wireless systems. However, they will not overcome the baseline power consumption of active base stations [13]. This makes it still attractive to power down systems when they are not loaded. The second group of approaches, new deployment strategies, adapt cell size to technology and traffic demands. However, these approaches do not adapt networks to the actually traffic load, for example, by following daily and weekly traffic profiles. This is done by the third group of approaches, system level management. These approaches not only overcome the above limitations of earlier research areas, but add their gains on top of them. For instance, even a very efficient base station can still save energy when it is switched to sleep mode. However, such network-wide adaptation adds high computational load and breaks the anytime-anywhere service paradigm of cellular access networks by generating coverage holes.

Many of the advancements in transmission and hardware technologies apply to a fully loaded network (i.e., during busy hour in dense urban areas), while real networks are often running with low efficiency at rather low load. Typical energy saving features implemented in commercial devices allow reducing resource utilization in these low load conditions; however, there is a nonnegligible power consumption offset that cannot be reduced unless the base station is switched to sleep state. The ideal energy behavior is a power consumption of the whole system that strongly depends on the traffic load, from a very low level with no traffic to a maximum value at full load. A network management that orchestrates base station activity and sleep periods can obtain this system behavior better than other local energy saving features. Unfortunately, energy efficiency was not taken into account when the cellular architecture was introduced. This generated severe constraints, which hamper the way towards this ideal behavior and, more in general, prevent reaching very large cuts of the energy consumption:

(i) The cellular architecture of wireless access networks has its foundation on the concept of full coverage of the service area that ensures that user terminals can get access to the network at all times in any point of the area.

(ii) Thus, a significant part of the network must be always on even without any traffic, resulting in unnecessary usage of resources, especially energy.

(iii) The data transmission is always coupled with the transmission of control signaling traffic (served by the same cell), although they have different characteristics.

It is straightforward to see that energy saving strategies in traditional cellular networks allow us mainly to exploit the sleep mode of redundant base stations in dense scenarios when the current traffic load is low. Studies in the field show that achievable energy savings in the current cellular architecture are in the range of $20 \%-40 \%$ [11], depending on the considered traffic profiles and network layouts, noting that a nonnegligible part of the network can never be switched to sleep mode, even if there are no active users.
In light of this, a new principle of mobile radio network energy-efficient architecture design, Beyond Cellular Green Generation $\left(\mathrm{BCG}^{2}\right)[14]$, was proposed in the framework of GreenTouch consortium, starting in January 2011. The basic idea of $\mathrm{BCG}^{2}$ is the concentration of control signaling in one so-called signaling layer (of a multilayered network), while data transmission is served by other layers/cells. Only the signaling cells are kept always on, while the data cells are available on demand. Despite not being a new concept in networking, the separation among signaling and data plane has represented a novelty for the wireless networking world. The results of $\mathrm{BCG}^{2}$ project have inspired several works in literature [15-19], which have extended application scenarios, considering technologies and models.

Similar approaches aiming more at a flexible resource management and signaling overhead reduction, rather than focusing on energy saving aspects have been proposed in $[20,21]$, where the idea of "phantom cell" has been presented. A separated architecture is proposed in [22] as well for a railway scenario in order to mitigate handover problems with high-speed trains. This solution is also considered in several main $5 \mathrm{G}$ projects, for example, MiWEBA (FP7-ICT EU-Japan Millimeter-Wave Evolution for Backhaul and Access (MiWEBA) project, http://www.miweba.eu), 5GrEEn (EIT-ICT Towards Green 5G Mobile Networks (5grEEn), http://wireless.kth.se/5green), MiWaveS (FP7-ICT EU Beyond 2020 Heterogeneous Wireless Network with Millimeter Wave Small Cell Access and Backhauling (MiWaveS) project, http://www.miwaves.eu), and standardization bodies, like in the study group of 3GGP on New Carrier Type.

In this paper, we discuss the $\mathrm{BCG}^{2}$ architecture and assess the energy efficiency improvement of mobile networks following the $\mathrm{BCG}^{2}$ concept. The main contributions of this paper are as follows. (1) We deeply analyze the limitations of the current cellular network approaches in reducing energy consumption and describe the advantages of a separated architecture. (2) We discuss technical challenges related to the implementation of the proposed architecture and open future research directions. (3) We design two stochastic models to assess the energy efficiency of realistic networks and evaluate the improvement when the $\mathrm{BCG}^{2}$ architecture is applied.

The remainder of the article is organized as follows. In Section 2, we highlight the limitations to energy management of current cellular networks. In Section 3, we describe the $\mathrm{BCG}^{2}$ and the characteristics that allow us to overcome current limitations. These characteristics introduce new technical challenges discussed in Section 4. In Section 5, we propose two stochastic models for computing network energy efficiency, while related results are shown in Section 6. We conclude the article with final remarks in Section 7.

\section{Limitations to Energy Management in Cellular Network}

Energy saving mechanisms are currently available in many commercial products. They are also one of the key features of the Self-Organizing Network (SON) which is being standardized by 3GPP for LTE [23]. The basic idea behind energy 
management strategies is that of exploiting the spare capacity available in cellular networks when traffic is low in order to put a subset of base stations in sleep mode. This is motivated by the typical energy consumption profile of base station equipment that is characterized by a remarkable difference between the minimum power of the active mode and the power of the sleep mode.

Improvements in the technologies and components of the base station equipment that are being investigated allow us to reduce the power consumption of all different types of devices, from femto-base stations to traditional macrobase stations. However, since the possible improvements on the hardware energy efficiency are limited by the baseline power consumption of active base stations, one has to recur to other means to achieve an energy profile of the network that is proportional to traffic load. Indeed, the ideal energy behavior we may wish from an energy management strategy is a power consumption of the whole system that is linearly dependent on the traffic load, and energy management strategies at system level are the only viable approach to achieve that. Reducing the maximum power consumption is obviously of paramount importance, but it is basically related to advancements in transmission and hardware technologies and not to network management. If we were able to achieve this ideal behavior at the network level, we could simply add any technology improvement to those of the energy management.

Unfortunately, there are some limiting constraints of the traditional cellular architecture that has been used so far for all wireless access technologies that prevent an optimal power management and, more in general, reach very high reductions of the energy consumption.

The cellular architecture of wireless access networks has its foundation on the concept of full coverage of the service area that ensures that user terminals in any point of the area can get access to the network at all times. The maximum coverage area that can be potentially covered by a base station depends on several issues, including the transmission power and the propagation conditions. Where traffic density is low, cell layouts commonly adopted are usually driven by the full coverage need and coverage ranges of base stations tend to be almost fully exploited by limiting the overlap among neighboring cells to what is necessary for the mobility management. In areas where traffic is much higher, like in crowded cities, base station density is highly increased in order to reduce cell size and increase the available access capacity per unit area. As a result, the cellular layout is characterized by a redundant coverage where each point in the service area is covered by several base stations.

It is quite evident that energy management procedures in traditional cellular architectures can only exploit, when traffic is low, the redundant, and overlapping, coverage of the network that is used to provide enough capacity when traffic is high. However, since full coverage must be ensured at all times, a nonnegligible part of the network can never be switched to sleep mode even if there is no active user.

It has been shown that potential energy savings achievable with energy management strategies in current cellular technologies are in the range of $20 \%-40 \%$, depending on the considered traffic profiles and network layouts [11]. Paradoxically, these savings may even reduce (in percentage) in the future with cellular architectures based on microcells. Indeed, it is commonly accepted that these microcellular layouts can reduce the nominal consumption since the energy per covered area of micro-base stations is lower than that of macro-base stations. Unfortunately, since microcells provide high capacity with limited coverage overlap, they leave little room for energy management since basically all cells are essential for guaranteeing full coverage. Even considering multilayer approaches and HetNet deployments, we can identify the same issue in the layer mainly used for coverage.

Therefore, the ideal behavior cannot be achieved with the traditional cellular architecture of current wireless access systems. We argue that this requires a paradigm shift that is able to completely reshape the structure of the network and provide revolutionary ways of ensuring ubiquitous wireless access to communication networks.

\section{Separated Design of Signaling and Data Transmissions}

The $\mathrm{BCG}^{2}$ paradigm shift is grounded on two key points. First, the mobile network must provide full-time and ubiquitous connectivity in a flexible way in order to allow a high degree of adaptation of base stations modes (including sleep modes). Not much information needs to be transmitted in order to enable ubiquitous connectivity. Basically, one has only to provide a signaling service to allow users to request a channel, when it is desired, and, in the opposite direction, to enable mobile paging. Second, signaling traffic is radically different from data traffic; therefore, sharing the same network architecture and the same radio interface is not optimal in terms of energy consumption. Indeed, the separation of data and signaling networks provides better energy efficiency management because of two important advantages. First, radio interfaces for the signaling network can be carefully designed for long-range low-rate transmissions. Current wireless broadband access interfaces are typically designed to provide high data rates within relatively short distances, for example, small cells in HetNet scenario or mmwave access technologies; they cannot efficiently support such transmissions. Therefore, the separation increases the energy efficiency since signaling radio interfaces can be optimized on a specific target and not according to the current data and signaling mix. Second, base stations for the data networks can be switched to sleep mode when there are no users with active data session under their coverage. As soon as a user becomes active, he can communicate his request to the signaling base station and the system can provide data connectivity by assigning it to an already active data base station or else by turning on a data base station that can serve the user. Ideally, the user is illuminated with a data service "spotlight" only where and when it is needed, as shown in Figure 1.

As mentioned above, the typical power profile of a base station is characterized by a power consumption that depends on the traffic load; however a nonnegligible amount of power is required even when the base station is serving no user. This 


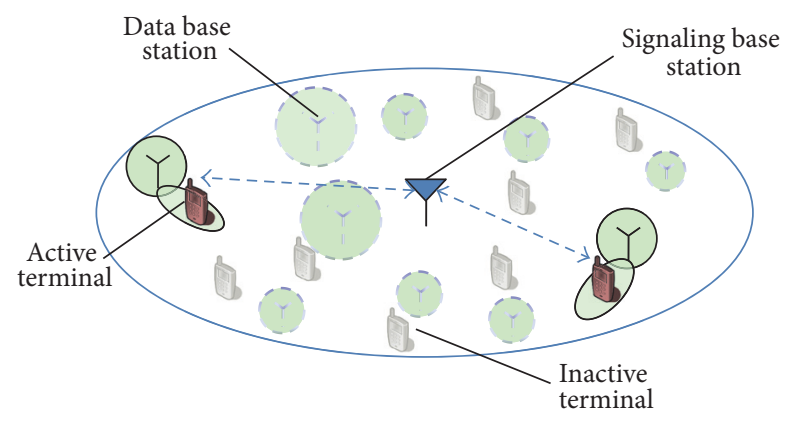

Figure 1: BCG ${ }^{2}$ scenario.

behavior is unavoidable, it characterizes current technology, and it will affect future devices as well, as reported in [24]. In order to reduce this energy consumption step, base stations must enter into a sleep mode, whose depth determines both the energy saving and the wake-up time. Results in [24] show that significant energy savings can be obtained with reasonably short wake-up times. Therefore, as suggested in Figure 2, the set of always-active base stations cause a noload step in the power profile of the whole network. The split architecture proposed in $\mathrm{BCG}^{2}$ project enables a system level network management able to get an almost load-proportional power profile of the whole network by dynamically activating data base stations according to user arrivals and departures. Advancements in transmission and hardware technologies can be easily integrated in the system in order to reduce the maximum power consumption.

Signaling cells are envisioned to be the only "always on" part of the network; they form a relatively "light-weight" overlay layer, leaner and more agile than in legacy HetNet networks. The signaling network must be dimensioned to just control signaling communications, possibly plus limited amount of, for example, extremely urgent or small data traffic. This provides a narrower traffic variation with respect to serving the whole data traffic demand, which translates in a better operation point for devices (especially of power amplifiers), with higher energy efficiency. A further benefit is the reduction of signaling overhead in the data network, particularly at low and medium load. A separated signaling network reduces the fixed signaling load and hence interference in data networks, which can be optimized for pure datadelivery performance.

We believe that this way of thinking wireless networks is the only viable approach to allow significant reduction on the energy consumption of networks as a whole and still be able to support high quality of service requirements. Clearly, data and signaling separation is not new in the networking literature; however in cellular networks it brings very important consequences, which lead to a considerable number of technical challenges. They arise from the interaction between signaling and data networks that calls for a renovated research effort in mobile networks, which will be briefly discussed in the following paragraphs.

\section{Technical Challenges}

The $\mathrm{BCG}^{2}$ architecture allows us to completely change the management strategy of mobile access networks. This has several advantages in terms of energy performance mainly related to the flexibility in the use of communication resources and in the activation of network elements only when they are needed. Moreover, additional advantages come from the opportunity to exploit heterogeneous data network with different wireless technologies and from a more efficient management of mobility even in the presence of system layouts with very small cells.

However, the implementation of this brand new architecture requires a research effort to tackle several technical challenges on different issues of $\mathrm{BCG}^{2}$ networks. In the following we point out and comment on these challenges and, whenever relevant, discuss how they are related to the additional advantages mentioned above.

4.1. Resource Management. Resource management is a key functionality in traditional wireless architectures, as it is crucial to select and allocate the radio resources necessary to serve a user request at the base station where the request was issued. In a new architecture where control and data planes may refer to different base stations, the complexity of resource management grows: the control plane has now a much larger set of options to choose from, as well as multiple objectives, such as connection quality, energy consumption, and resource utilization. Even selecting the best base station to serve a new user is a challenge.

The resource selection procedures in $\mathrm{BCG}^{2}$ architecture have to include the selection of the base station that will serve a service request and the management of the interaction between the signaling network and the data network. These procedures are a key component of the $\mathrm{BCG}^{2}$ architecture to reduce the energy consumption since they determine the energy state of network devices. Resource selection needs to take into account not only the context information on the service request, but also the status of the network and their devices. Generally speaking, the selection and (if required) activation of a base station able to serve a user request given the context information discussed above are not a difficult task. Unfortunately, optimizing this decision process is much more complicated. Even the identification of an optimization objective is not easy, due to the obvious tradeoff between energy cost and performance and the number of constraints involved for quality of service requirements, terminal capability limitations, technologies of base stations in range, and available radio resources.

Resource management is naturally an online decision process and the incremental energy cost for the assignment of a service request to a base station depends on its status. If the base station is already active the incremental cost for serving and additional user is usually much smaller than in the case when it is in sleep mode and needs to be reactivated. When mobility is an important issue of the user context, algorithms for resource activation and management need to orchestrate the selection of a sequence of base stations following the movement of the user. Clearly, the availability of context 


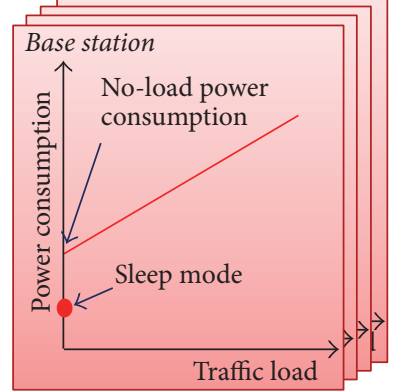

Sum of individual contributions

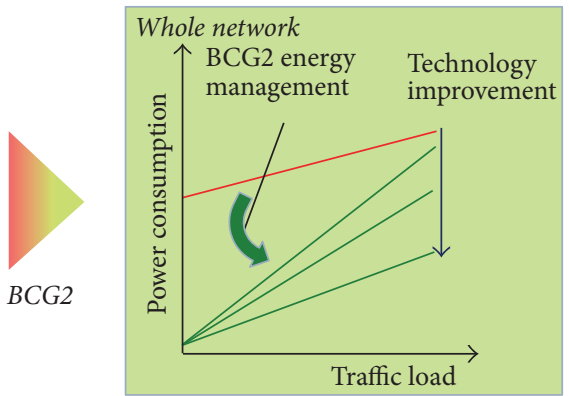

Orchestration of the whole network

FIgURE 2: $\mathrm{BCG}^{2}$ rationale.

information at the control plane is crucial to the resource allocation process and is much more important here than in traditional architectures.

4.2. Context Awareness. Traditional signaling mechanisms adopted by current cellular technologies interact with user terminals to get information on the service requests and with the network elements necessary to provide the service. The separation of data and signaling functions complicates the gathering of context information, like channel quality. Network-related measurements typically executed on the transmissions of signaling symbols cannot be used to estimate either the availability or the quality of data communication channels, since these are provided by different base stations. Channel quality information may not be retrievable even with direct scanning of reference signals from potential serving base stations, as they could be inactive due to the energy saving policies or unreachable due to difficult signal propagation or directive antenna techniques. Therefore, in this scenario, basic information on channel quality estimation for wireless access must be provided through the context and often estimated using indirect measures [25].

The signaling network of $\mathrm{BCG}^{2}$ needs to get more information from the users; this richer information is required in order to characterize what can be called the "context" of a service request. The context is the key element that allows the signaling network to activate appropriate resource selection algorithms for the data network.

Context information can be divided into two basic groups: network context and user context. The network context is the set of information that describes the status of the network, for example, type and position of devices, activity status, energy consumption, capacity and current load. Such information is operator-related and easily gathered via the backhauling/core network. Thus, the network context is generally assumed to be perfectly known to the resource allocation entity. The user context needed to efficiently run the resource allocation includes both the information on user mobility and service requirements.

A first and fundamental user-context information to be additionally included in the context is the user terminal location with respect to the access points or base stations that can potentially serve its request. This is obviously not necessary in traditional systems since the base station that gets the service request is the same that serves it. The location service used for estimating user terminal position can be part of the functionalities provided by the new signaling network or be provided by external systems. Several localization systems can be also used in parallel in order to obtaine the best possible estimation to be used by the mobile network when necessary, according to scenario (e.g., outdoor or indoor) and services supported by the terminal (e.g., satellite positioning).

Not only the location of user terminal at the moment of the service request but also its estimated mobility can be an important element to characterize the context. Information on user mobility can help to identify the base station to activate, avoiding, for instance, selecting a cell that with high probability will be quickly left by the terminal during its movement. During data session, mobility estimation and user context can be used to track user movements and make predictions that can be used to take resource reallocation decisions (including handovers) in advance to compensate for delays caused by base station activation or technology switch.

In addition to location and channel quality, other pieces of information can help the resource selection process of the new $\mathrm{BCG}^{2}$ architecture, such as terminal capabilities (supported data network technologies, transmission rates, power, etc.) and user profile (service level agreed with the provider, required quality of service, preferences on different access options, user category, etc.).

4.3. Handover Management. The separate architecture provides a new playground wherein handover policies can be optimized. First, traditional handovers based on RRC Connection Reconfiguration will be less frequent. Indeed, a connection with a long-range signaling base stations will be always active while users move among data base stations. Second, users will monitor their connection quality with surrounding data base stations and send this information uplink through the signaling connection. According to this information, together with context information, the network resource allocation system will select candidate base stations to hand over the connection, which must be informed and prepared in advance. The work in [26] presents a detailed overview of the signaling message exchanges in a network where control and user plane are split over two network 
layers. Differently from the legacy systems, these base stations may be inactive; therefore, the system must decide which base station to switch on and, depending on the user mobility, whether the base station will be ready when the user will need to make the change. Third, the strong emphasis on energy efficiency aspects opens new opportunities to be investigated in handover scenarios. For instance, if the best performancewise destination of a handover is inactive, the system may decide to opt for another suboptimal base station to keep the best base station inactive and save energy. Otherwise, if the requested service is not throughput intensive, the user may even be served by the signaling base station itself, breaking the symmetry of the system to have a further energy saving benefit. Fourth, the opportunity of having an extremely flexible handover management and to resort to long-range signaling cells will clearly impact the network planning strategies for handover-related aspects.

4.4. Assessment of $B C G^{2}$ Advantages and Performance. Analyzing and estimating the potential savings provided by the new network architecture in terms of energy consumption are one of the first technical challenges to be tackled. Taking a closer look at the advantages allowed by the $\mathrm{BCG}^{2}$ architecture, it is easy to see that they are basically twofold. As mentioned before, the new mobile network architecture clearly enables the fine-tuning of the network operating conditions according to a generic daily traffic profile. But, in addition, it also allows following different daily traffic profiles in different locations of the coverage area. For instance, regions with high density of business activities usually experience a traffic peak during the morning, while residential areas show hightraffic levels during the evening, when people go back home. Obviously, the mobile network is dimensioned for supporting peaks even if they are not contemporary and some spare capacity is always present. The proposed architecture is able to exploit spare capacity to reduce energy consumption since traffic peaks are both spatially and temporally separated.

The goal of works addressing this challenge would be that of showing, through models, mathematical analysis, and simulations based on realistic data and traffic distributions, which could be the theoretical limits of the $\mathrm{BCG}^{2}$ approach and which could be the bounds on the energy saving performance in such limit conditions. Then, an analysis of the expected performance in realistic deployments must be carried out considering relevant scenarios. This analysis could be targeted on assessing energy saving in low-traffic condition and evaluating how the system, designed to save energy, behaves in high-traffic scenarios, in order to highlight possible criticalities.

4.5. System Architecture. As shown in the previous section, the core idea of $\mathrm{BCG}^{2}$ is the separation of data and signaling infrastructure. Signaling devices will be able, of course, to monitor and control data network devices; however, defining the architecture components and how they are organized in detail is one of the toughest challenges. Two types of approaches can be adopted: a hierarchical architecture where the computational intelligence is centralized and located far from the access network or a flat architecture where each device is equipped with computational power.

A possible solution in favor of the flat architecture, strongly advocated by the LTE standards, opens a further future research scenario. Given the considerable processing power that will be available at each access point, $\mathrm{BCG}^{2}$ algorithms can be directly designed to exploit this feature and engineered according to a distributed computing paradigm. In this case, it would be interesting to investigate which functions could be implemented in a distributed manner, which ones should be centralized, and which level of selforganization the $\mathrm{BCG}^{2}$ architecture can reach. An interesting possibility is that of exploiting cloud-computing approaches for the computational heavy tasks that can be remotely executed.

4.6. Heterogeneous Data Networks. The basic concept of the $\mathrm{BCG}^{2}$ architecture is independent of the wireless technology adopted for the data network even if only future generation low-power wireless technologies will allow us to achieve very high energy efficiency that is the main target of the new architecture. But more than that, it is worth pointing out that $\mathrm{BCG}^{2}$ enables a flexible management of a set of heterogeneous data networks using different wireless technologies.

The lifecycle of wireless technologies is much longer than it was expected. A high fraction of mobile voice calls is still handled by the old GSM technology in many countries. Most of mobile handhelds have today multiple radio interfaces with different technologies and even base station equipment is currently being replaced with multitechnology devices attached to a single radio access network. Dealing with the coexistence of heterogeneous wireless technologies is somehow unavoidable and it is commonly recognized as a key element for the design of future generation mobile network architectures.

$\mathrm{BCG}^{2}$ architecture is particularly suitable for managing heterogeneous wireless technologies as it reverses the classical approach to network selection. Since the access to communication service is mediated by the signaling network, it is no longer the user terminal that selects the access point, but this is basically delegated to the network. This allows a more flexible and intelligent management of traffic with the set of technologies and radio resources available through algorithms that are able to take into account the status of the whole system. The design of these new algorithms is an interesting technical challenge since several issues can be considered including specific resource management policies of mobile operators. Mobility management among different data networks during active communication sessions is also an important aspect of the problem with, however, the advantage of the new system architecture to be in full control of resource selection.

4.7. Radio Technologies for the Signaling Access. Traditional cellular systems have been designed in order to operate at high data rates. However, the signaling network proposed here for $\mathrm{BCG}^{2}$ has completely different characteristics, such as robustness, low energy consumption, long range, and low data rates. Therefore, the design of such network requires 
a paradigm shift from the traditional approaches and it is probably one of the hardest among these technical challenges.

As for radio spectrum resources, the need to create relative large coverage areas for signaling base stations suggests using low frequencies that have better propagation conditions and obstacle penetration. Regulation authorities at international level are currently reorganizing spectrum assignments taking advantage of the dismissal of some old radio technologies like analog TV. This is making some small bands below $1 \mathrm{GHz}$ available that can be suitable for a low-rate network like the one needed for $\mathrm{BCG}^{2}$.

Several multiple access technologies can be adopted for the signaling network. However the need to minimize energy consumption tends to privilege time based access scheme that allows a better power management according to traffic level. Multiple antenna technologies can also be exploited to improve energy performance and, if necessary, to support the localization service for the context information.

At architecture level, several solutions are possible, ranging from extreme scenarios where all signaling functionalities for the management of data sessions are implemented in the new overlay signaling network to hybrid cases where only session setup is managed by the signaling network, while session control is delegated to data network. In any case, the integration among control functions in the data networks and the signaling network and energy consumption of the signaling networks are fundamental part of the system design that needs to be addressed.

As far as the mobility management is concerned, the signaling network offers new opportunities to improve efficiency. It is worth mentioning here that this is particularly relevant in the presence of network layouts with very small cells that are able to provide very high broadband access capacity per unit area and energy efficiency. With traditional technologies the main limiting factor that prevents using very small cells is obviously mobility management that requires quick handover procedures. With a separated signaling network, performance requirements on handover procedures can be relaxed since decisions can be taken in advance and communicated to user terminal via a robust radio channel.

4.8. Network Planning. From the network planning perspective the optimization of the geographic location of both data and signaling infrastructures is an important topic to be worked on. Such optimization has to take into account the interoperability of the two infrastructures and to maximize the flexibility of the network. Furthermore, the planning has to account for the spatial-temporal load profile throughout the network. Hence, some degree of overprovisioning of the signaling network is desirable in order to allow network adaptation with respect to the exploited context (e.g., user, application, and load profile) and to guarantee the network flexibility required for power management mechanisms. Nevertheless, the costs involved in overprovisioning constraint the network optimization problem. An efficient network design must not only take into account the energy management but also jointly consider both deployment and operational costs.

Since many possible alternatives must be made available in the planning process, in addition to the already complex network scenario depicted above, we expect that heuristics and simulations have to be applied for the joint optimization of the data network with the signaling radio and network architecture.

A final remark is on the concept of coverage. Traditionally, in the weakest definition of coverage, a user is defined to be covered if it can receive the pilot signal of at least one cell with sufficient strength. The $\mathrm{BCG}^{2}$ architecture opens room for more extreme coverage definition where a user can be defined covered if it can receive a transmission from a signaling access point within a given time interval and communicate with at least one data access point upon request. This may lead to addressing the problem of designing optimized activity patterns for signaling network devices, which can bring additional energy savings.

\section{Performance Models}

In order to understand the potential energy saving provided by the radical architectural shift of the $\mathrm{BCG}^{2}$ approach, we have developed two statistical models. They focus on data base stations (DBSs), since we believe signaling base stations will have a limited impact on the total energy consumption of $\mathrm{BCG}^{2}$ network.

Both models assume Mobile Terminals (MTs) distributed over an area according to a 2D Poisson process with density $\lambda_{\mathrm{MT}}$. Data base stations deployment is assumed to be 2D Poisson distributed as well, with density $\lambda_{\mathrm{BS}}$, or, equivalently, with average intersite distance is $\mathrm{d}_{\mathrm{BS}}$. We consider a unique and homogeneous network layer characterized by a single value of is $d_{\mathrm{BS}}$ or $\lambda_{\mathrm{BS}}$. The goal of these models is to compute the DBS activation probability $\beta$, which can be interpreted as the percentage of the number of active DBSs with respect to the total number of deployed ones or, alternatively, the percentage of the time a DBS is active. The first model, named Poisson model, considers the service area of each DBS AS (show in Figure 3(a)), which can be approximately computed using the hexagonal-cell model (note that we use the hexagonal-cell model only to derive an approximate value of the cell area starting from the intersite distance. The Poisson model does not rely on hexagonal deployment; it only needs the average cell area $A_{s}$ ) as $A_{s} \cong$ $(\sqrt{3} / 2)$ is $\mathrm{d}_{\mathrm{BS}}^{2}$; therefore the probability $\beta$ can be computed as the probability that at least one MT is located in the service area of a given DBS:

$$
\beta=1-e^{\lambda_{\mathrm{MT}} A_{s}} \text {. }
$$

The second model, named Integral Geometry model, leverages the fact that the coverage area of each DBS, namely, the area where MT can decode transmissions from DBS, is larger than the service area, the area where DBS reference signal is received as the strongest. In this case, the overlap with adjacent cells can be used to save energy. Indeed, a DBS can be put in sleep mode either when no user is in its service area or when users in its service area can be covered by a neighboring active DBS, thanks to the coverage area overlap. This overlap effect can be modeled using Integral Geometry techniques. 


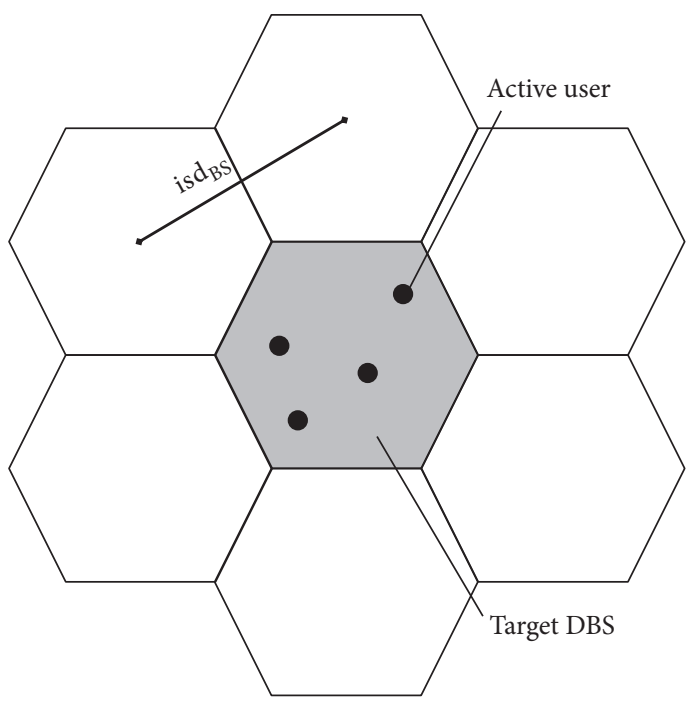

(a) Poisson model

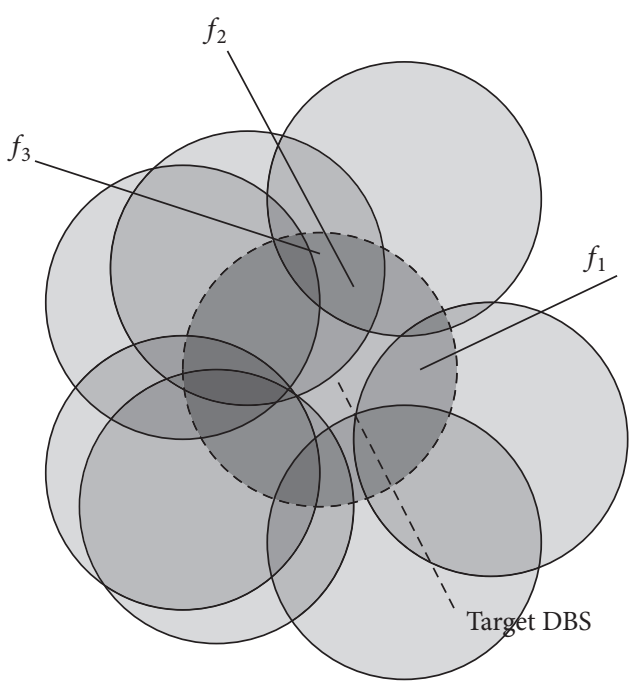

(b) Integral Geometry model

FIGURE 3: Examples of the two analytical models.

We consider a "target" DBS with circular coverage area $A_{C}$, radius $R_{C}$, and perimeter $P_{C}$. This DBS can have $N$ adjacent DBSs whose coverage areas intersect the target coverage area, as shown in Figure 3(b). The DBSs are randomly distributed, as explained at the beginning of the section, with coverage area, radius, and perimeter equal to the ones of the target DBS.

The fraction $f_{k} \in[0,1]$ of the coverage area of the target DBS covered by exactly $k$ adjacent DBSs can be computed using Integral Geometry [27] as follows:

$$
f_{k}=\frac{\left(\begin{array}{c}
N \\
k
\end{array}\right)\left(2 \pi A_{C}\right)^{k}\left(2 \pi A_{C}+P_{C}^{2}\right)^{N-k}}{\left(2 \pi\left(2 A_{C}\right)+P_{C}^{2}\right)^{N}} .
$$

Note that this value does not depend on the shape of the coverage area, provided it is convex, but only on its area and perimeter. Using (2), the probability $P_{k}$ that at least a user lies in the fraction of target coverage area which is covered by $k$ adjacent DBSs can be computed, similarly to the first model, as follows:

$$
P_{k}=1-e^{\lambda_{\mathrm{MT}} f_{k} A_{\mathrm{C}}} .
$$

The final activation probability $\beta$ can be computed by solving the following equation, where we applied the assumption of homogeneous scenario, which is equivalent to force the activation probability to be the same for every DBS:

$$
\begin{aligned}
\beta & =P_{0} \\
\cdots & +\left(1-P_{0}\right) P_{1}(1-\beta) \\
\cdots & +\left(1-P_{0}\right)\left(1-P_{1}\right) P_{2}(1-\beta)^{2} \\
\cdots & +\cdots \\
& =P_{0}+\left(1-P_{0}\right) P_{1}(1-\beta) \\
& +\left(1-P_{0}\right) \sum_{i=2}^{N}\left(\prod_{n=1}^{i-1}\left(1-P_{n}\right)\right) P_{i}(1-\beta)^{i} .
\end{aligned}
$$

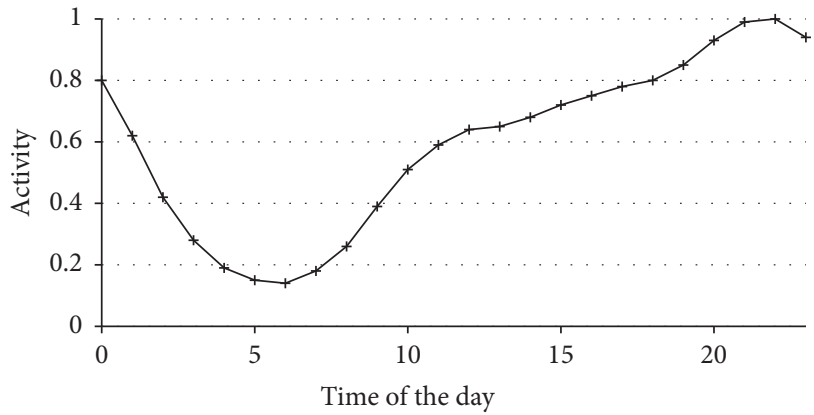

Figure 4: Daily data traffic profile.

Formula in (4) computes the DBS activation probability as the sum of the probabilities of the following disjoint events: there is at least one user in the fraction of the coverage area not covered by any adjacent DBS (first line); there are no users in the area covered by no adjacent DBS, but there is at least one user in the area covered by one adjacent DBS and the adjacent DBS is in sleep mode (second line); there are no users both in the area covered by no adjacent DBS and in the area covered by 1 adjacent DBS, but there is at least one user in the area covered by 2 adjacent DBSs and both DBSs are in sleep mode (third line); and so forth.

\section{Numerical Results}

The accuracy of the models and the achievable energy saving with $\mathrm{BCG}^{2}$ approach have been tested in the following scenario. We have considered the typical daily data traffic profile in Figure 4 [2], while DBS types and relative parameters are shown in Table 1; they are derived from the study in [2].

We have analyzed the potential energy saving within three network setups which are characterized by a traffic 
TABLE 1: Data base station types and relative parameters [2].

\begin{tabular}{lccc}
\hline DBS type & Macro & Micro & Pico \\
\hline isd $_{\mathrm{BS}}[\mathrm{m}]$ & 500 & 150 & 50 \\
$\lambda_{\mathrm{BS}}\left[\mathrm{DBS} / \mathrm{km}^{2}\right]$ & 14 & 51 & 462 \\
ON power $[\mathrm{W}]$ & 1000.0 & 144.6 & 14.7 \\
Sleep power $[\mathrm{W}]$ & 0.1 & 0.1 & 0.1 \\
Range $[\mathrm{m}]$ & 1500 & 1000 & 400 \\
\hline
\end{tabular}

level and a network configuration which can be assumed to be similar to the 2010 ones, 2015 ones, and 2020 predictions. Namely, we have considered a low-traffic area with density $6 \mathrm{Mbps} / \mathrm{km}^{2}$ served by macro-DBSs (2010 scenario), a mid-traffic area with density $30 \mathrm{Mbps} / \mathrm{km}^{2}$ served $40 \%$ by macro-DBSs, and $60 \%$ by micro-DBSs (2015 scenario) and high-traffic area with density $120 \mathrm{Mbps} / \mathrm{km}^{2}$ served $10 \%$ by macro-DBSs, $60 \%$ by micro-DBSs, and $30 \%$ by picoDBSs (2020 scenario). We fix the per-user traffic demand equal to $2 \mathrm{Mbps}$ in order to convert traffic densities in [2] to reasonable user densities.

In order to verify the accuracy of the model, we have compared the model predictions against results from a Monte Carlo simulator. The simulator generates several instances of the service area by deploying DBSs and MTs according to the given distributions. A covering heuristic assigns MTs to the smallest number of DBSs as possible, and then, unloaded DBSs are put in sleep mode. This simulates a network scheduler that aggregates load in few DBSs. The activation probability is the average ratio among active and deployed DBSs. The instance generation and the heuristic algorithm are repeated for each time of the day in the profile of Figure 4.

Figure 5 shows the daily traffic profile plotted along with the activation probabilities computed with the models and the Monte Carlo simulator. Note how the overlap among cells allows switching to sleep mode many DBSs, making the Poisson model overestimate the practical activation probability computed by the simulator.

The gap between the results of the Integral Geometry model and the ones of the simulator is mainly due to the active DBS selection process. While in the Integral Geometry model the activation probabilities among nearby DBSs are assumed to be independent (see (4)), in the Monte Carlo simulator they are not. Indeed, since the simulator includes an optimization phase to minimize the number of active DBSs, the activation probabilities of DBSs close to already active DBSs are smaller, in particular when the traffic is low. Nevertheless, the Integral Geometry model appears to give a correct, although conservative, estimation of the behavior of the $\mathrm{BCG}^{2}$ network. The results from both models over 2015 and 2020 scenarios are shown in Figure 6. In particular, it shows that the gap reduces when the number of deployed base stations increases.

To evaluate the energy saving of $\mathrm{BCG}^{2}$ networks with respect to legacy systems in different scenarios and with different traffic levels, we use energy-per-bit [Joule/bit] as energy efficiency performance figure. This value can be computed as the ratio between the energy required by DBSs in their

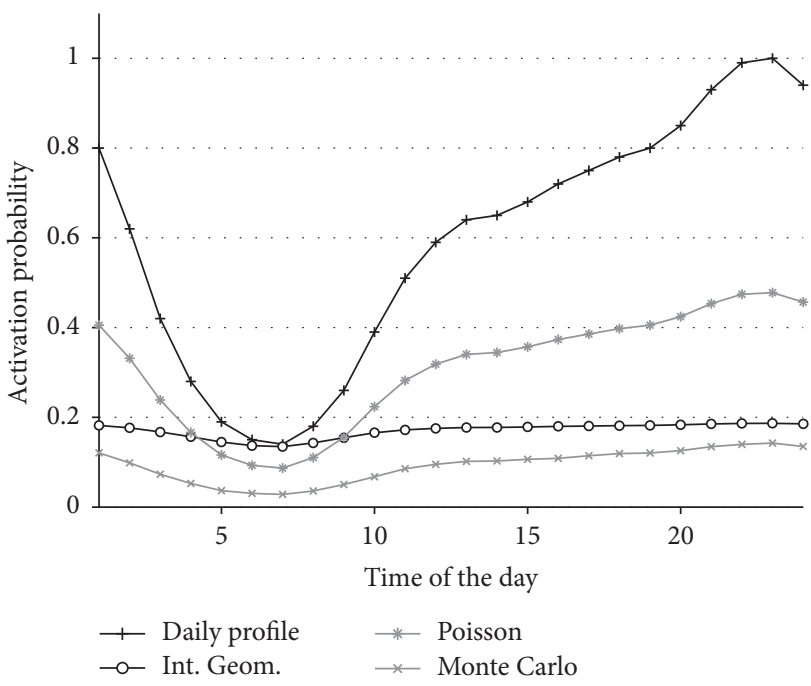

FIGURE 5: Daily traffic profile plotted against DBS activation probabilities in 2010 scenario.

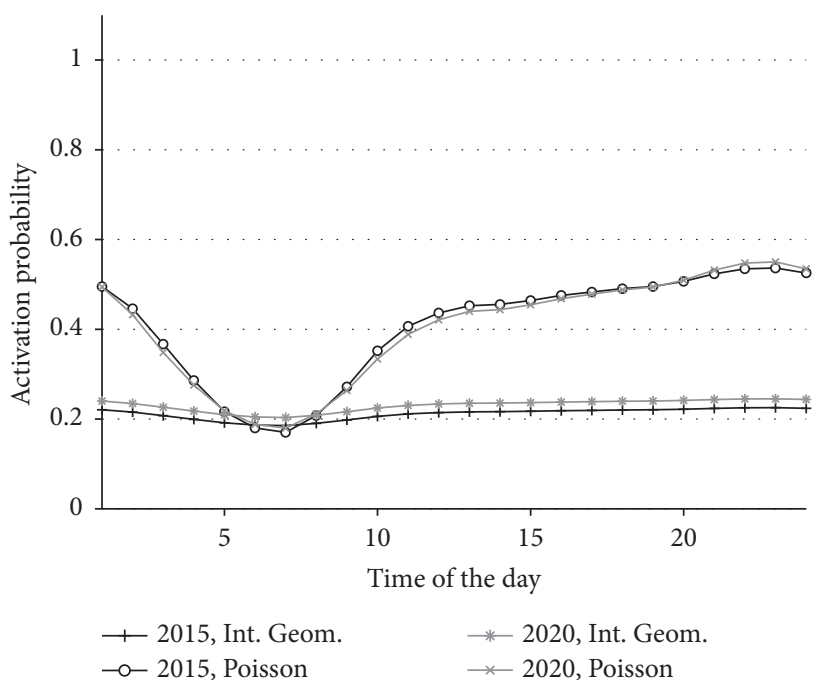

FIGURE 6: DBS activation probabilities for 2015 and 2020 scenarios.

TABLE 2: Energy efficiency values for legacy and BCG $^{2}$ cellular networks.

\begin{tabular}{lccc}
\hline Efficiency [J/MBit] & 2010 & 2015 & 2020 \\
\hline Legacy & 1295.6 & 353.44 & 97.49 \\
BCG $^{2}$ & 221.75 & 75.23 & 22.73 \\
\hline
\end{tabular}

activity for the entire day and the total amount of traffic volume served within the same day.

Table 2 shows achievable energy efficiencies with both $\mathrm{BCG}^{2}$ and legacy approaches. $\mathrm{BCG}^{2}$ energy efficiencies have been computed using the Integral Geometry model, while legacy energy efficiencies consider all the deployed DBSs always active.

The $\mathrm{BCG}^{2}$ approach allows achieving much higher energy efficiencies, reducing the amount of energy needed to 
exchange information bits. Compared to the current situation, legacy 2010 scenario, $\mathrm{BCG}^{2}$ networks can potentially lead to a 2020 scenario where cellular networks can be operated more than 50 times more efficiently. The use of $\mathrm{BCG}^{2}$ networks in the 2020 scenario allows us to achieve an energy efficiency more than four times higher than the one with the legacy approach.

\section{Conclusions}

This paper introduced the ideas investigated in the $\mathrm{BCG}^{2}$ project of the GreenTouch consortium. The basic concept is the separation of signaling and data in the network. The independent signaling network is a promising solution that provides freedom for data transmission adaptation. This freedom comes from the fact that the data access points only have to cover areas with active users since the signaling network guarantees full coverage of the system. This solution is very energy efficient since the data access points do not waste transmitted power on areas without active users.

We believe that this way of thinking wireless networks is one of the most promising approaches to achieve a significant reduction on the energy consumption of networks as a whole and still be able to cope with high quality of service requirements. We have analyzed its potential energy saving over traditional approaches throughout analytical models.

Several technical challenges were identified as well. Context management, resource management, signaling radio interface design, and network planning strategies are some of the interesting research directions opened by this network paradigm.

\section{Competing Interests}

The authors declare that there is no conflict of interests regarding the publication of this paper.

\section{Acknowledgments}

This article is an extension of the conference paper [14]: the authors would like to thank Dr. Dos Santos and Dr. Gloss for that initial discussion, which stimulated their effort to extend the work into this journal article.

\section{References}

[1] D. Ferling, T. Bitzer, T. Bohn, D. Wiegner, and A. Pascht, "Power efficient transceivers to enable energy-efficient mobile radio systems," Bell Labs Technical Journal, vol. 15, no. 2, pp. 59-76, 2010.

[2] M. Imran and E. Katranaras, "Energy efficiency analysis of the reference systems, areas of improvements and target breakdown. ict-earth project, deliverable d2. 3, ec-ist office, brussels, belgium (january 2011)".

[3] D. Tipper, A. Rezgui, P. Krishnamurthy, and P. Pacharintanakul, "Dimming cellular networks," in Proceedings of the IEEE Global Communications Conference (GLOBECOM '10), December 2010.
[4] F. Richter and G. Fettweis, "Cellular mobile network densification utilizing micro base stations," in Proceedings of the IEEE International Conference on Communications (ICC '10), Cape Town, South Africa, May 2010.

[5] H. Karl, "An overview of energy-efficiency techniques for mobile communication systems," Report of AG Mobikom WG7, 2003.

[6] M. Savi, O. Ayoub, F. Musumeci, Z. Li, G. Verticale, and M. Tornatore, "Energy-efficient caching for video-on-demand in fixed-mobile convergent networks," in Proceedings of the IEEE Online Conference on Green Communications (OnlineGreenComm '15), pp. 17-22, IEEE, Piscataway, NJ, USA, November 2015.

[7] K. Dufková, M. Bjelica, B. Moon, L. Kencl, and J.-Y. Le Boudéc, "Energy savings for cellular network with evaluation of impact on data traffic performance," in Proceedings of the European Wireless Conference (EW '10), pp. 916-923, IEEE, Lucca, Italy, April 2010.

[8] R. Giuliano, F. Mazzenga, and M. Petracca, "Energy efficient planning of cellular radio networks," in Proceedings of the 24th Tyrrhenian International Workshop on Digital CommunicationsGreen Information and Communications Technology (TIWDC '13), Genoa, Italy, September 2013.

[9] S. Sarkar, R. K. Ganti, and M. Haenggi, "Optimal base station density for power efficiency in cellular networks," in Proceedings of the 1st IEEE International Conference on Communications (ICC '14), pp. 4054-4059, Sydney, Australia, June 2014.

[10] J. Lorincz, A. Capone, and D. Begušić, "Optimized network management for energy savings of wireless access networks," Computer Networks, vol. 55, no. 3, pp. 514-540, 2011.

[11] M. A. Marsan, L. Chiaraviglio, D. Ciullo, and M. Meo, "Optimal energy savings in cellular access networks," in Proceedings of the IEEE International Conference on Communications Workshops (ICC '09), June 2009.

[12] L. Chiaraviglio, D. Ciullo, M. Meo, and M. A. Marsan, "Energyefficient management of UMTS access networks," in Proceedings of the 21st International Teletraffic Congress (ITC 21 '09): Traffic and Performance Issues in Networks of the Future-Final Programme, September 2009.

[13] A. Capone, S. D’Elia, I. Filippini, and M. Zangani, "Measurement-based energy consumption profiling of mobile radio networks," in Proceedings of the 1st IEEE International Forum on Research and Technologies for Society and Industry (RTSI '15), pp. 127-131, Torino, Italy, September 2015.

[14] A. Capone, A. F. Dos Santos, I. Filippini, and B. Gloss, "Looking beyond green cellular networks," in Proceedings of the 9th Annual Conference on Wireless On-Demand Network Systems and Services (WONS '12), pp. 127-130, January 2012.

[15] A. Zakrzewska, D. López-Pérez, S. Kucera, and H. Claussen, "Dual connectivity in LTE HetNets with split control- and userplane," in Proceedings of the IEEE Globecom Workshops (GC Wkshps '13), pp. 391-396, IEEE, Atlanta, Ga, USA, December 2013.

[16] X. Xu, G. He, S. Zhang, Y. Chen, and S. Xu, "On functionality separation for green mobile networks: concept study over LTE," IEEE Communications Magazine, vol. 51, no. 5, pp. 82-90, 2013.

[17] J. Wu, S. Zhou, Z. Niu, C. Liu, P. Yang, and G. Miao, “Trafficaware data and signaling resource management for green cellular networks," in Proceedings of the 1st IEEE International Conference on Communications (ICC '14), pp. 3499-3504, Sydney, Australia, June 2014. 
[18] Z. Wang and W. Zhang, "A separation architecture for achieving energy-efficient cellular networking," IEEE Transactions on Wireless Communications, vol. 13, no. 6, pp. 3113-3123, 2014.

[19] J. Zhang, X. Zhang, C. Liu et al., "Theoretical study and performance evaluation of macro-assisted data-only carrier for next generation $5 \mathrm{~g}$ system," International Journal of Communication Systems, vol. 30, no. 2, 2017.

[20] S. Liu, J. Wu, C. H. Koh, and V. K. N. Lau, "A 25 Gb/s(/km2) Urban wireless network beyond IMT-advanced," IEEE Communications Magazine, vol. 49, no. 2, pp. 122-129, 2011.

[21] Y. Kishiyama, A. Benjebbour, T. Nakamura, and H. Ishii, "Future steps of LTE-A: evolution toward integration of local area and wide area systems," IEEE Wireless Communications, vol. 20, no. 1, pp. 12-18, 2013.

[22] L. Yan and X. Fang, "Reliability evaluation of 5G C/U-plane decoupled architecture for high-speed railway," EURASIP Journal on Wireless Communications and Networking, vol. 2014, article no. 127, 2014.

[23] U. Barth and E. Kuehn, "Self-organization in 4G mobile networks: Motivation and vision," in Proceedings of the 7th International Symposium on Wireless Communication Systems (ISWCS '10), pp. 731-735, York, UK, September 2010.

[24] B. Debaillie, C. Desset, and F. Louagie, "A flexible and futureproof power model for cellular base stations," in Proceedings of the 81st IEEE Vehicular Technology Conference (VTC Spring '15), Glasgow, United Kingdom, May 2015.

[25] A. Redondi, I. Filippini, and A. Capone, "Context management in energy-efficient radio access networks," in Proceedings of the 24th Tyrrhenian International Workshop on Digital Communications-Green ICT (TIWDC '13), pp. 1-5, Genoa Italy, September 2013.

[26] H. Song, X. Fang, and L. Yan, "Handover scheme for 5G C/U plane split heterogeneous network in high-speed railway," IEEE Transactions on Vehicular Technology, vol. 63, no. 9, pp. 46334646, 2014.

[27] L. Lazos and R. Poovendran, "Stochastic coverage in heterogeneous sensor networks," ACM Transactions on Sensor Networks, vol. 2, no. 3, pp. 325-358, 2006. 

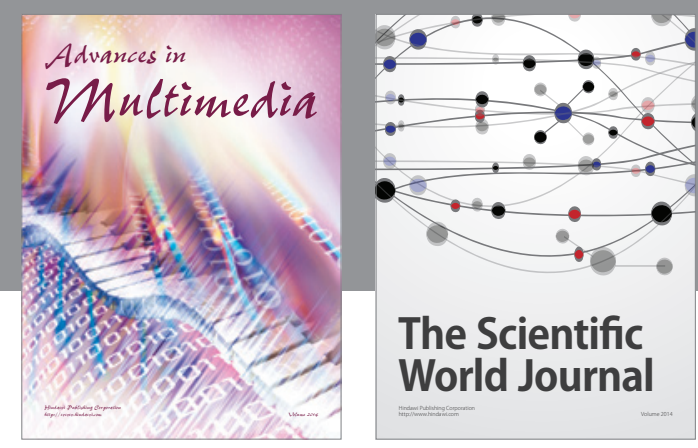

The Scientific World Journal
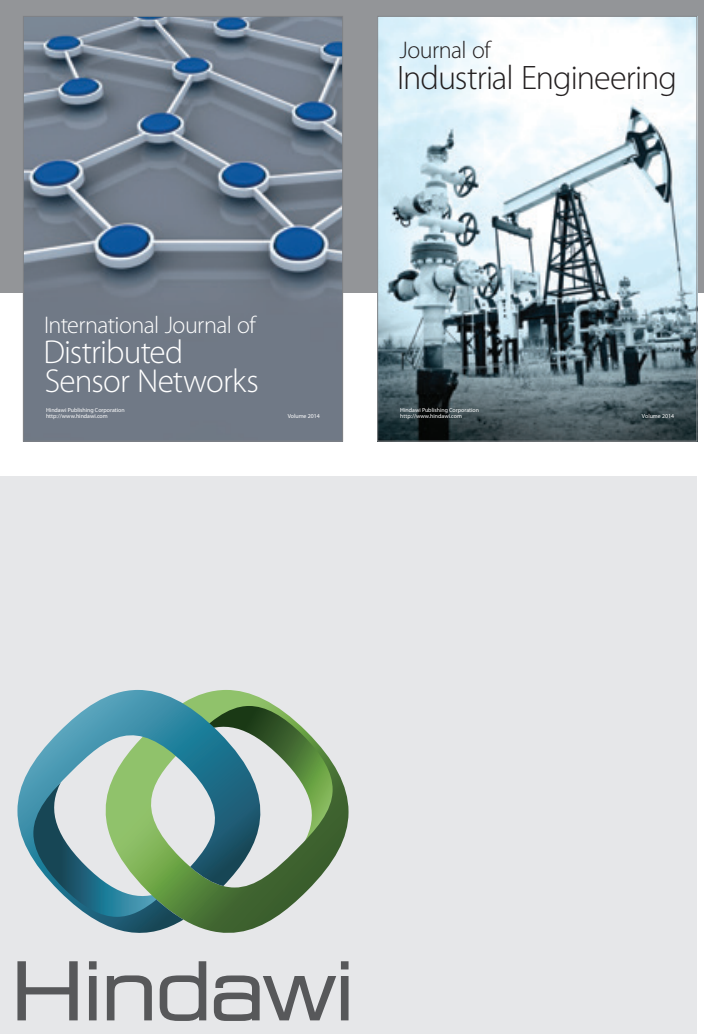

Submit your manuscripts at

https://www.hindawi.com

\section{Computer Networks} and Communications
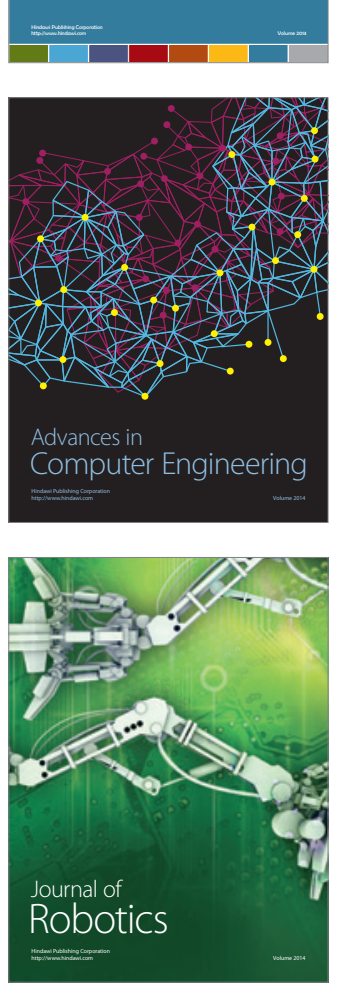
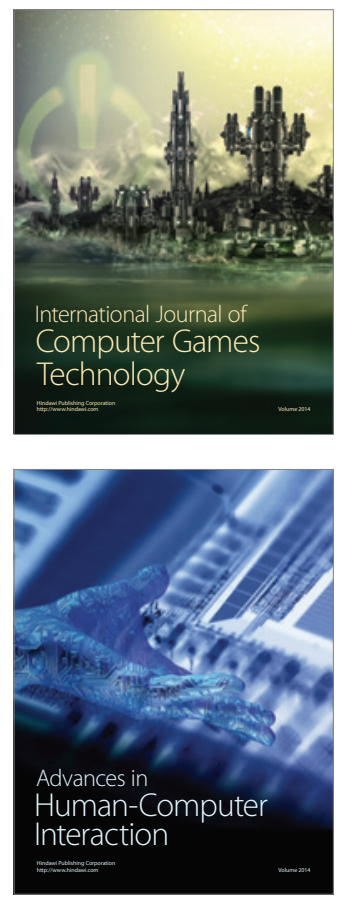
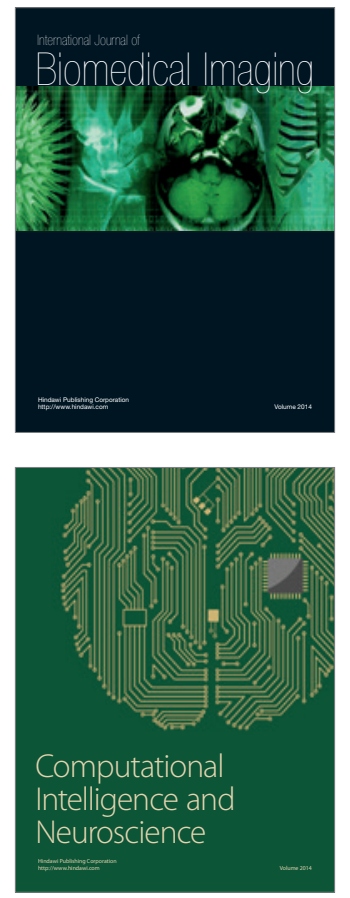
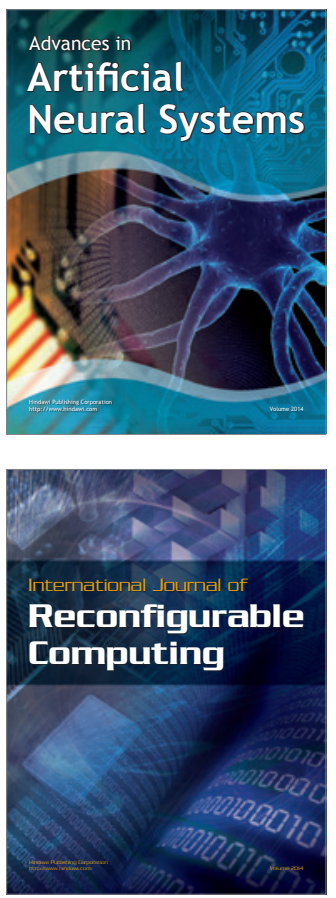
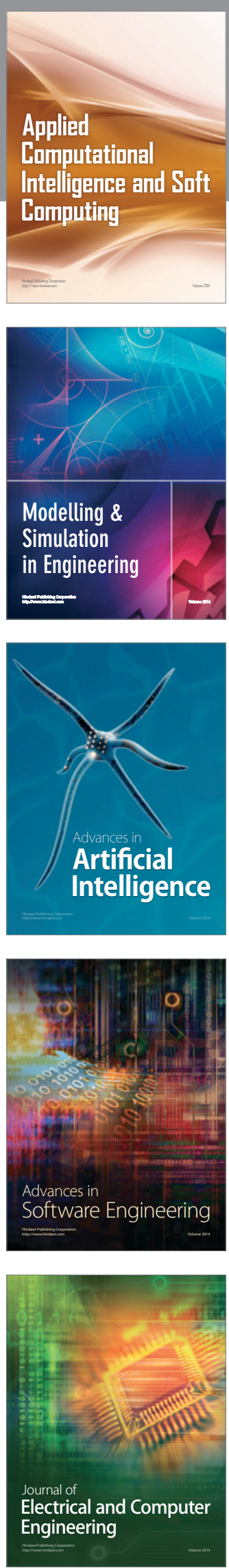